\title{
Electrical peripheral nerve stimulation relieves bone cancer pain by inducing Arc protein expression in the spinal cord dorsal horn
}

This article was published in the following Dove Press journal: Journal of Pain Research

\author{
Ke-fu Sun ${ }^{1-3}$ \\ Wan-wen Feng ${ }^{2,3}$ \\ Yue-peng $\mathrm{Liu}^{4}$ \\ Yan-bin Dong ${ }^{4}$ \\ Li Gao 2,3 \\ Hui-lin Yang' \\ 'Department of Orthopedic \\ Surgery, the First Affiliated Hospital \\ of Soochow University, Suzhou, \\ Jiangsu, China; ${ }^{2}$ Department of \\ Orthopedics, Lianyungang Oriental \\ Hospital, Lianyungang, Jiangsu, \\ China; ${ }^{3}$ Department of Orthopedics, \\ Affiliated Lianyungang Oriental \\ Hospital of Xuzhou Medical \\ University, Lianyungang, Jiangsu, China; \\ ${ }^{4}$ Center for Clinical Research and \\ Translation Medicine, Lianyungang \\ Oriental Hospital, Lianyungang, \\ Jiangsu, China
}

\begin{abstract}
Objective: The analgesic effect on chronic pain of peripheral nerve stimulation (PNS) has been proven, but its underlying mechanism remains unknown. Therefore, this study aimed to assess the analgesic effect of PNS on bone cancer pain in a rat model and to explore the underlying mechanism.
\end{abstract}

Materials and methods: PNS on sciatic nerves with bipolar electrode was performed in both naïve and bone cancer pain model rats. Then, the protein levels of activity-regulated cytoskeleton-associated protein (Arc), $\alpha$-amino-3-hydroxy-5-methyl-4-isoxazolepropionic acid-type glutamate receptor 1 (GluA1), and phosphate $N$-methyl-D-aspartic acid-type glutamate receptor subunit $2 \mathrm{~B}$ (pGluNR2B) in spinal cord were evaluated by immunohistochemistry and Western blotting. Thermal paw withdraw latency and mechanical paw withdraw threshold were used to estimate the analgesic effect of PNS on bone cancer pain. Intrathecal administration of Arc shRNA was used to inhibit Arc expression in the spinal cord.

Results: PNS at 60 and $120 \mathrm{~Hz}$ for 20 min overtly induced Arc expression in the spinal cord, increased thermal pain thresholds in naïve rats, and relieved bone cancer pain; meanwhile, $10 \mathrm{~Hz}$ PNS did not achieve those results. In addition, PNS at 60 and $120 \mathrm{~Hz}$ also reduced the expression of GluA1, but not pGluNR2B, in the spinal cord. Finally, the anti-nociceptive effect and GluA1 downregulation induced by PNS were inhibited by intrathecal administration of Arc shRNA.

Conclusion: PNS ( $60 \mathrm{~Hz}, 0.3 \mathrm{~mA}$ ) can relieve bone-cancer-induced allodynia and hyperalgesia by upregulating Arc protein expression and then by decreasing GluA1 transcription in the spinal cord dorsal horn.

Keywords: peripheral nerve stimulation, Arc, GluA1, bone cancer pain

\section{Introduction}

Bone cancer pain induced by bone metastasis or original bone cancer severely decreases patients' quality of life. ${ }^{1}$ About $50 \%$ of patients suffer this "severe" pain ${ }^{1,2}$ that is described as "dreadful" by the Visual Analogue Scale, a widely used pain assessment tool. ${ }^{3}$ In addition, bone cancer pain cannot be controlled by common treatment, such as surgery and drugs. Opioids are effective but they often cause some side effects. ${ }^{4}$ Therefore, it is urgent to develop new methods for treating bone cancer pain. ${ }^{1,2}$

Many electrical nerve stimulation methods can relieve pain, such as TENS, ${ }^{5} \mathrm{PENS},{ }^{6}$ and PNS. ${ }^{7,8}$ Among them, PNS is effective in relieving neuropathic pain, ${ }^{8}$ diabetic pain, ${ }^{9}$ and chronic headache,${ }^{10}$ but the best strategy for using PNS for intractable pain is under exploration. In fact, PNS at $40 \mathrm{~Hz}$ with a pulse width of 300-450 ms reduces $60 \%$ severity of trigeminal neuropathic pain, ${ }^{11}$ at $50 \mathrm{~Hz}(250 \mu \mathrm{s}$ of width, $0.15-0.3 \mathrm{~V}$ of amplitude) relieves chronic post-traumatic neuropathic pain by $>75 \%,{ }^{12}$ and at $50 \mathrm{~Hz}$
Correspondence: Hui-lin Yang Department of Orthopaedic Surgery, the First Affiliated Hospital of Soochow University, No.899 Pinghai Road, Suzhou, Jiangsu 215006, China Email huilinyangsuda@।63.com 
$(1 \mathrm{~mA}, 50 \mu \mathrm{s})$ treats post-amputation pain effectively. ${ }^{13}$ Taken together, PNS at $40-100 \mathrm{~Hz}$ and intensity lower than that used in TENS or PENS is an available strategy for relieving intractable pain. Understanding the underlying mechanism can help improve the treatment effectiveness for intractable pain. The gate control theory proposed by Melzack and Wall may explain how activating large myelinated nerve fibers by PNS decreases pain levels by inhibiting the transmission of pain signals (and "close the gate") from the spinal cord to higher centers in the central nervous system. ${ }^{14}$ In addition, PNS could change the concentrations of several neurotransmitters in the spinal cord, including GABA, ${ }^{15}$ serotonin, and dopamine. ${ }^{16}$

Like c-fos and Zif268, Arc, also known as $\operatorname{arg3.1,}$ belongs to a family containing many types of early-response genes. ${ }^{17}$ One characteristic of Arc is that it can be induced by peripheral electrical nerve stimulation. Indeed, high frequency $(>100 \mathrm{~Hz})$, but not low frequency $(<10 \mathrm{~Hz})$, currents are effective in inducing Arc transcription and translation. ${ }^{18}$ Another characteristic is that Arc mRNA can move to dendrites and reduce the density of AMPAR on dendrite membrane by internalizing AMPAR. ${ }^{17-19}$ It is also reported that Arc regulates global GluA1 levels in nucleus. ${ }^{20}$ Whatever be the case, since AMPAR participates in the process of neuropathic and inflammatory and bone cancer pain,,$^{21,22}$ it is hypothesized that Arc might negatively regulate chronic pain $^{23}$ by internalizing AMPAR or by decreasing AMPAR expression.

This evidence inspires us to unveil the mechanism underlying the anti-nociceptive effects of PNS, TENS, PENS, and even SCS. We hypothesized that PNS could induce Arc expression in the spinal cord and then reduce the density of membrane AMPAR by promoting AMPAR internalization on dendrites and/or decrease global AMPAR expression, which together results in pain relief. This study aimed to assess the effect of PNS on bone cancer pain and explore the role of Arc in the process.

\section{Materials and methods}

\section{Animals and drugs}

Female adult Sprague-Dawley rats (7-8 weeks; initial weight: $160-180 \mathrm{~g}$ ) were supplied by Xuzhou Medical College and housed in a controlled 12-hour dark/light cycle with free access to food and water. Menstrual cycle was not synchronized as it will not alter the development of bone cancer pain. ${ }^{24} \mathrm{~A}$ total of 254 rats were used in this experiment; the experiment was approved by the Institutional Animal Care and Use Committee of Lianyungang Oriental Hospital and conducted in accordance with the Declaration of the National Institutes of Health Guide for Care and Use of Laboratory Animals. To knock down gene expression, $5 \mu \mathrm{L}$ of Arc shRNA lentiviral particles (Santa Cruz Biotechnology Inc., Dallas, TX, USA) containing three target-specific constructs that encode 19-25 nt (plus hairpin) shRNAs was intrathecally administered 1 day before PNS.

\section{Bone cancer pain animal model}

Walker 256 mammary gland carcinoma cells were supplied by Dr Song, Neuroscience Research Institute, Beijing Medical University. The use of gifted cells was approved by the institutional review board of the Key Lab of Anesthesiology of Xuzhou Medical University. Before surgery, tumor cells $\left(1 \times 10^{5}\right.$ cells $\left./ \mu \mathrm{L}\right)$ were obtained from ascetic tumor-bearing female rats and kept on ice before injection. Rats were anesthetized by intraperitoneally injecting sodium pentobarbital $(50 \mathrm{mg} / \mathrm{kg})$. After shaving and disinfecting with $70 \%(\mathrm{v} / \mathrm{v})$ ethanol, a skin incision parallel to the tibia was made to expose the tibial plateau. Tumor cells $\left(1 \times 10^{5}\right.$ cells/ $\mu \mathrm{L}, 5 \mu \mathrm{L}$ ) or boiled cells (sham group) were injected into the intramedullary space of the right tibia. The injection site was closed with bone wax immediately after syringe removal. The incision was then dusted with penicillin powder and closed.

\section{Radiographic observation}

On the 14th day after TCI, radiographic images were acquired (exposure setting: $12 \mathrm{~ms}, 31 \mathrm{KVp}$ ) using Philips Digital Radiographer System (Digital Diagnost VM; Philips Medical Systems DMC GmbH, Hamburg, Germany). The destruction of bone was scored on a scale of 0-5: 0 , normal bone structure without any sign of deterioration; 1 , small radiolucent lesions in the proximal epiphysis $(<3)$; 2 , increased number of radiolucent lesions ( $>3$ ) indicating loss of medullary bone; 3 , loss of medullary bone and erosion of the cortical bone; 4 , full-thickness unicortical bone loss; 5, full-thickness bicortical bone loss and displaced fracture. ${ }^{24}$

\section{Histology}

Histological examination of the affected bone was carried out on all 14 days after TCI. Rats were anesthetized and transcardially perfused with $0.9 \%$ saline and $4 \%$ paraformaldehyde. Then, tibia specimens were removed and demineralized in $15 \%$ ethylenediaminetetraacetic acid solution with a supersonic device (HD-350; Huida Devices Company, Hubei, China) until they could be penetrated easily by a needle. Sections of $10 \mu \mathrm{m}$ were obtained with a cryomicrotome and stained with H-E. 


\section{PNS}

A device (JL-C4-V2a; Jialong Educational Devices Company, Shanghai, China) with bipolar electrode was used for PNS. Combined anesthesia was used during electric stimulation. Sodium pentobarbital $(25 \mathrm{mg} / \mathrm{kg}$, intraperitonially) was firstly applied as basic anesthesia, and then used $2 \%$ isoflurane to maintain anesthesia through a mask. The frequency of respiration is maintained one to two times per second as appropriate anesthesia depth. From the branching point of the sciatic nerve at the middle part of the femur, 1 $\mathrm{cm}$ was exposed and stimulated with a bipolar electrode using square waves of 10,60 , and $120 \mathrm{~Hz}$ frequencies $(0.5 \mathrm{~ms}$ and $0.3 \mathrm{~mA}$ for $20 \mathrm{~min})$. Current intensity $(0.3 \mathrm{~mA})$ was chosen according to previous studies ${ }^{11,12}$ and the preliminary experiment. Current of $0.6 \mathrm{~mA}$ was also tested in the preliminary experiment, but it was so strong that it "burned" the tissues around nerves. Therefore, $0.3 \mathrm{~mA}$ current was adopted. Current intensity was monitored with an ampere meter during the stimulation. When the stimulation was completed, the incision was sutured with 4-0 silk thread. The animals were sacrificed 2 hours after PNS, and samples were collected for Western blotting and immunohistochemistry.

\section{Behavioral tests}

Thermal hyperalgesia was indicated by a significantly shortened latency of paw withdrawal in response to heat stimulation. To determine thermal hyperalgesia, a radiant heat source (model 390, series 8; IITC Inc., Woodland Hills, CA, USA) was focused and delivered on the middle part of the plantar surface just behind the last toe, with the thermal stimuli shut off automatically when the hind paw moved (immediately or 20 seconds later) to escape tissue damage. Thermal stimuli were delivered three times to each hind paw at an interval of 5-8 min. Two different intensities of radiant heat were adopted: $50 \%$ radiant heat intensity with a baseline of 6-to8 -second thermal withdrawal latency was used to detect the effect of PNS on normal pain threshold and $45 \%$ radiant heat intensity with a 13-to-15-second baseline of thermal withdrawal latency was used to detect the effect of PNS on bone cancer pain.

Mechanical allodynia was reflected by a significant decrease in the threshold of paw withdrawal to mechanical indentation on the plantar surface of each hind paw by a sharp, cylindrical probe. To increase the force gradually, the probe (38540 electronic von Frey; Ugo Basile, Varese, Italy) was applied to the middle part of the plantar surface just behind the last toe. A 30 second interval was set between tests. The minimal force inducing paw with- drawal was read off the display. Average values from six tests were considered in the final analysis. The threshold of mechanical withdrawal of each animal was converted into milli-Newtons $(\mathrm{mN})(\mathrm{mN}=9.8 \mathrm{~N} / \mathrm{kg})$. The experiments were single blinded.

\section{Western blotting}

Western blotting was used to quantify the protein levels of Arc, GluA1, and $N$-methyl-D-aspartic acid receptor 2B subunit. L4-L5 DRG and/or spinal cord segments were quickly removed from deeply anesthetized rats and stored at $-80^{\circ} \mathrm{C}$. Tissue samples were lysed in ice-cold $\left(4^{\circ} \mathrm{C}\right) \mathrm{NP}-40$ lysis buffer containing a cocktail of protease inhibitors, phosphatase inhibitors, and phenylmethylsulfonyl fluoride (Sigma-Aldrich, St Louis, MO, USA). Protein concentrations in the lysates were estimated by the bicinchoninic acid method (Beyotime, Nantong, China). Equal amounts of total protein from each sample were separated by sodium dodecyl sulfate polyacrylamide gel electrophoresis and transferred onto $0.2 \mu \mathrm{m}$ polyvinylidene difluoride membranes (Millipore, Bedford, MA, USA). The following primary antibodies were used: anti-Arc (1:2000; Abcam, Cambridge, UK), antiglutamate receptor 1 (1:1000; Millipore), anti-pGluNR2B (Tyr1472) (1:1000; Millipore), and anti-GAPDH (1:5000 10,000; Sigma-Aldrich). The membranes were developed by enhanced chemiluminescence reagents (Thermo Fisher Scientific, Waltham, MA, USA) after incubation with horseradish peroxidase-conjugated secondary antibodies (Santa Cruz Biotechnology Inc.). Data were analyzed using built-in package of image software (NIH, Washington, DC, USA).

\section{Immunohistochemistry}

Deeply anesthetized rats were sequentially and transcardially perfused with $0.9 \%$ saline and $4 \%$ formaldehyde. L4-L5 spinal cord segments were removed and post-fixed in $4 \%$ formaldehyde overnight. Sections $(15 \mu \mathrm{m})$ were cut using a cryomicrotome (CM1950; Leica Instrument Gmbh, Nussloch, Germany). For immunochemistry, free-floating sections were blocked in PBS containing 5\% donkey serum or $5 \%$ goat serum according to host species of the secondary antibody for 2 hours and incubated with primary antibodies at $4^{\circ} \mathrm{C}$ overnight. The sections were then washed with $0.01 \mathrm{M}$ PBS ( $\mathrm{pH} 7.4 ; 3 \times 5 \mathrm{~min}$ ) followed by incubation with secondary antibodies at room temperature for 2 hours. The sections were developed using the 3-3 -diaminobenzidine reagent (Beyotime) and mounted with $90 \%$ glycerin for microscopic observation. The dilutions of antibodies were as follows: anti-Arc (1:200; Abcam) and anti-Glutamate receptor 1 (1:100; Millipore). 


\section{Statistical analysis}

Data are presented as mean \pm standard error of the mean or median \pm median absolute deviation. Statistical analyses were carried out with Statistical Product and Service Solutions, version 15.0 (SPSS Inc., Chicago, IL, USA). Data normality was assessed by the Shapiro-Wilk test and variance equality was evaluated by Levene's test. When the data was normal and equally variance, parametric analysis of variance (ANOVA) was used; otherwise, Mann-Whitney $U$ test was used. Two-way repeated-measures ANOVA (days $\times$ groups) was used to assess behavioral responses to thermal and mechanical stimuli, followed by Bonferroni post hoc tests. The non-parametric statistical method was used to analyze bone radiographs, quantity of protein, and histological observations. $P<0.05$ was considered statistically significant.

\section{Results}

\section{Establishment of a bone cancer pain model}

X-ray radiography taken on the 14th day after TCI showed that a part of the tibia became thin (Figure 1C), and bone

A

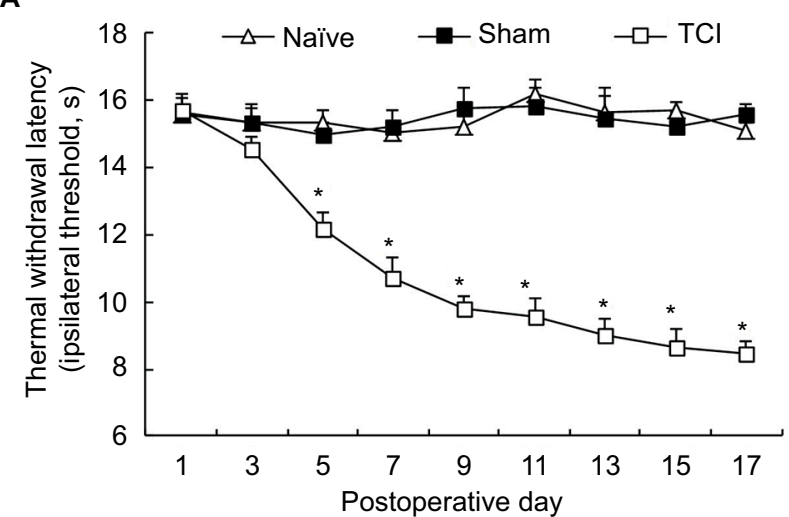

C

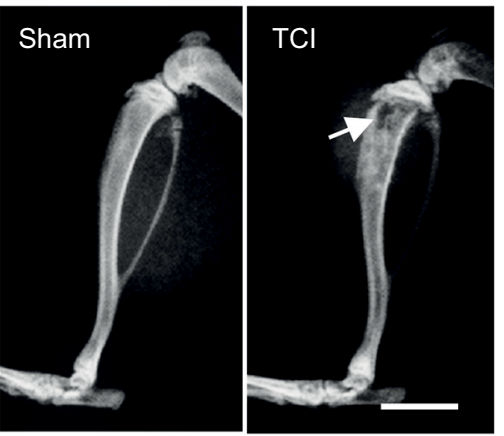

D

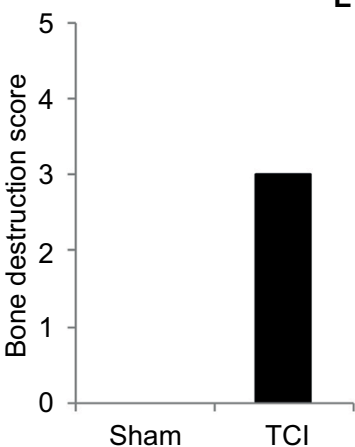

destruction score was identified as level 3 (Figure 1D); $\mathrm{H}-\mathrm{E}$ (Figure 1E) staining further revealed that the inner surface of the bone was deteriorated by tumor cells and became rough, with multiple "tunnels" in the bone cortex. PWTL (Figure 1A), which reflects hyperalgesia occurrence, decreased from day 3 after TCI to day 21; in addition, PWMT (Figure 1B), which assesses allodynia existence, had a similar trend to PWTL.

\section{Effect of PNS at different frequencies on pain thresholds and Arc, GluAI, and pGluNR2B expression in naïve animals}

For naïve animals, PNS at $60 \mathrm{~Hz}$ and $120 \mathrm{~Hz}$ increased thermal pain threshold by 2 and 3 days respectively (Figure 2A); meanwhile, PNS at the same frequency had no effect on mechanical pain threshold (Figure 2B). PNS at $10 \mathrm{~Hz}$ had no influence on the mechanical and thermal pain thresholds (Figure 2A and B). PNS at 60 and $120 \mathrm{~Hz}(0.3 \mathrm{~mA}, 20 \mathrm{~min}$ ) overtly induced Arc protein expression in spinal dorsal horn, but PNS at $10 \mathrm{~Hz}$ could not (Figure 2C and D). Immunohistochemistry was performed to explore Arc localization;
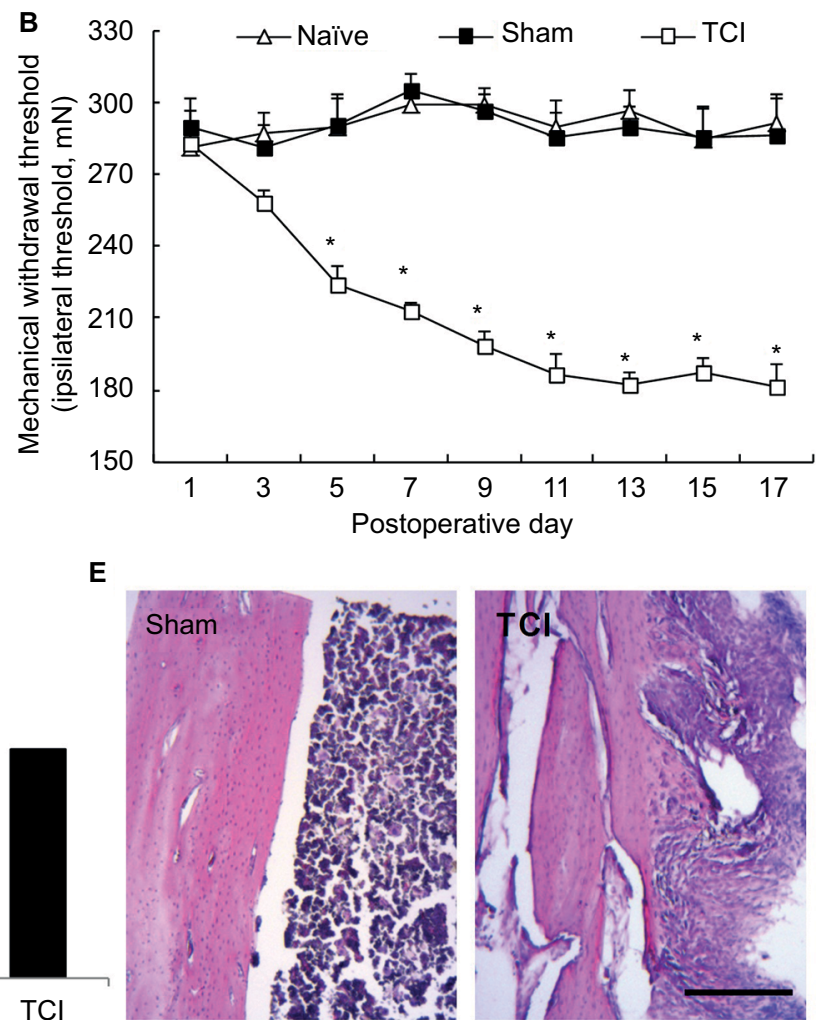

Figure I Walker 256 tumor cells invade bones and induce cancer pain.

Notes: (A) Thermal withdrawal latency (a sign of hyperalgesia) test shows that TCl induces hyperalgesia in female rats. (B) Mechanical withdrawal threshold (a sign of allodynia) shows that $\mathrm{TCl}$ induces allodynia in rats ( $\mathrm{n}$ of each group $=8$ ); the data are presented as mean \pm SEM and analyzed by two-way ANOVA. Compared with the sham rats, rats receiving $\mathrm{TCl}$ exhibit apparent bone invasion as detected by $\mathrm{X}$-ray $(\mathbf{C})$ and bone destruction score reaches 3 on the scale (D) (n of each group $=4$, data are expressed as median \pm MAD and analyzed by Mann-Whitney $U$ test; both MAD =0). (E) H-E stain ( $n$ of each group $=3$ ) shows that the inner surface of bone cavity has been deteriorated by tumor cells and multiple "tunnels" have formed in bone cortex. White arrow indicates thinning cortical bone that can be attributed to the invasion of tumor cells. Bars: $(\mathbf{C})=5 \mathrm{~mm} ;(\mathbf{E})=100 \mu \mathrm{m}$. $* P<0.05$, compared with sham.

Abbreviations: ANOVA, analysis of variance; SEM, standard error of the mean; MAD, median absolute deviation; $\mathrm{H}-\mathrm{E}$, hematoxylin and eosin; TCl, tumor cell implantation. 

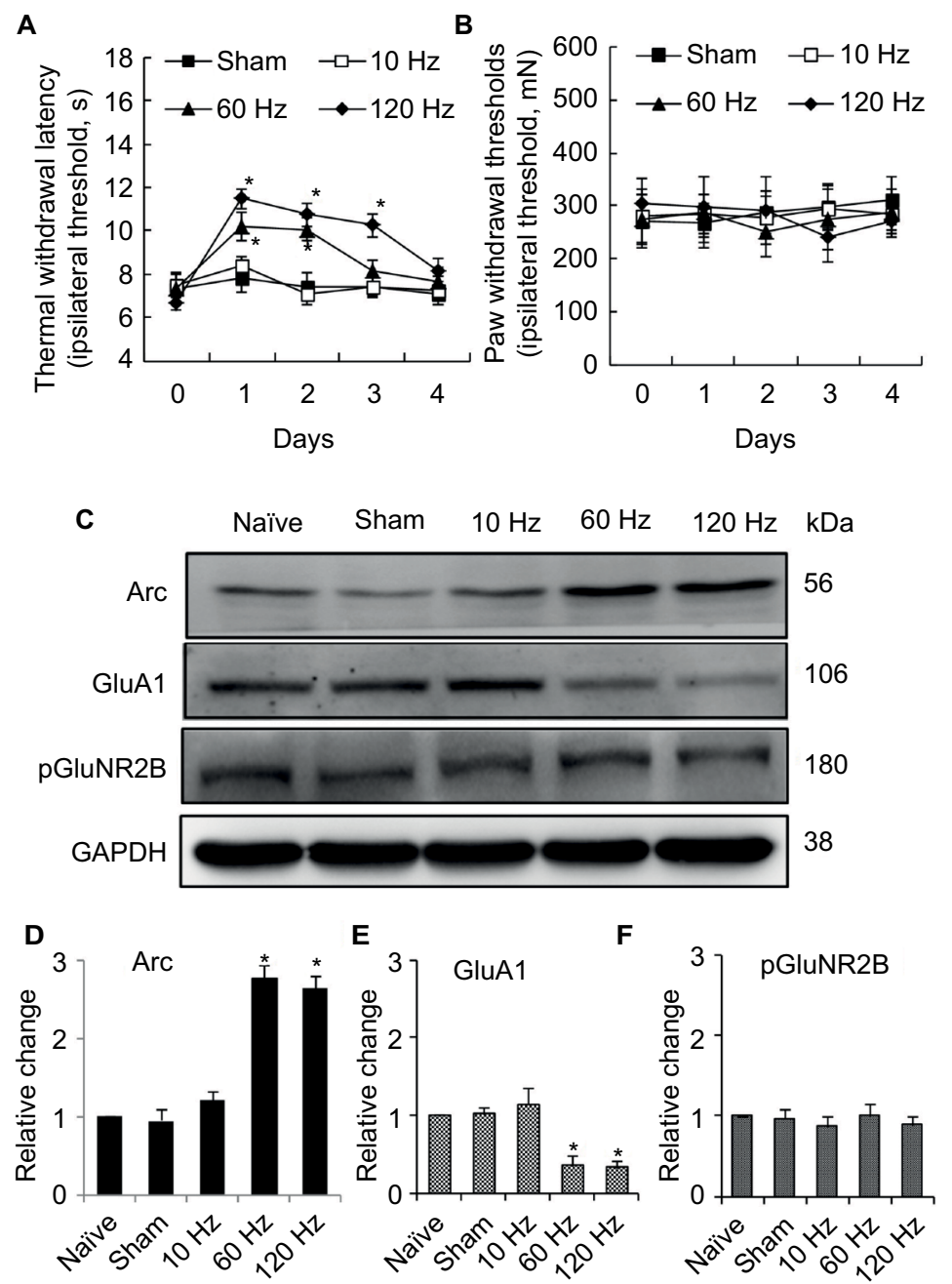

Figure 2 Effect of different frequency PNS on pain thresholds as well as expression of Arc, GluAI, and pGluNR2B in naïve rats.

Notes: PNS at $60 \mathrm{~Hz}$ and $120 \mathrm{~Hz}$ increases thermal pain thresholds, while $10 \mathrm{~Hz}$ PNS does not (A). PNS at all three frequencies did not influence mechanical thresholds (B) ( $\mathrm{n}$ of each group $=8$; ${ }^{*} \mathrm{P}<0.05$, compared with sham; data are presented as mean $\pm \mathrm{SEM}$ and analyzed with two-way ANOVA). Meanwhile, $60 \mathrm{~Hz}$ and I20 $\mathrm{Hz} \mathrm{PNS}$ induce Arc protein expression (C, D) and decrease GluAI expression (C, E) in the spinal cord, which has no effect on pGluNR2B (C, F), while $10 \mathrm{~Hz}$ PNS has no or minor effect on Arc expression or on the reduction of GluAI expression ( $n$ of each group $=5$; $* P<0.05$, compared with sham; data are presented as median \pm MAD and analyzed with Mann-Whitney $U$ test).

Abbreviations: ANOVA, analysis of variance; GluAI, $\alpha$-amino-3-hydroxy-5-methyl-4-isoxazolepropionic acid-type glutamate receptor I; pGluNR2B, phosphate N-methylD-aspartic acid-type glutamate receptor subunit 2B; PNS, peripheral nerve stimulation; SEM, standard error of the mean; MAD, median absolute deviation; Arc, activityregulated cytoskeleton-associated protein.

bipolar electrode induced Arc expression in lamina I-II of spinal cord dorsal horn (Figure 3). Meanwhile, PNS at 60 and $120 \mathrm{~Hz}$ also decreased GluA1 expression without affecting pGluNR2B levels (Figure 2C, E, and F).

\section{Effects of PNS at different frequencies on Arc, GluAI, and pGluNR2B expression in an animal model of bone cancer}

TCI induced the expression of Arc, GluA1, and pGluNR2B in the spinal cord on day 14. At this time, PNS at 60 and 120 $\mathrm{Hz}$ for $20 \mathrm{~min}$ further induced Arc expression and decreased the concentration of GluA1 in rats with bone cancer pain (Figure 4). Immunohistochemistry showed that GluA1 expression increased in the bone cancer pain animal model, and PNS negatively regulated increased GluA1 expression in the dorsal horn (Figure 5).

\section{Effects of Arc shRNA on anti-nociceptive properties of PNS, and Arc and GluAI expression, in bone cancer pain animal model}

In the bone cancer pain animal model, PNS at 60 and 120 $\mathrm{Hz}$ administrated on day 7 after TCI increased both PWTL and PWMT (Figure 6A and B); this anti-nociceptive effect lasted for nearly 6 days. When Arc shRNA was intrathecally administrated 1 day before PNS, the anti-nociceptive effect of 

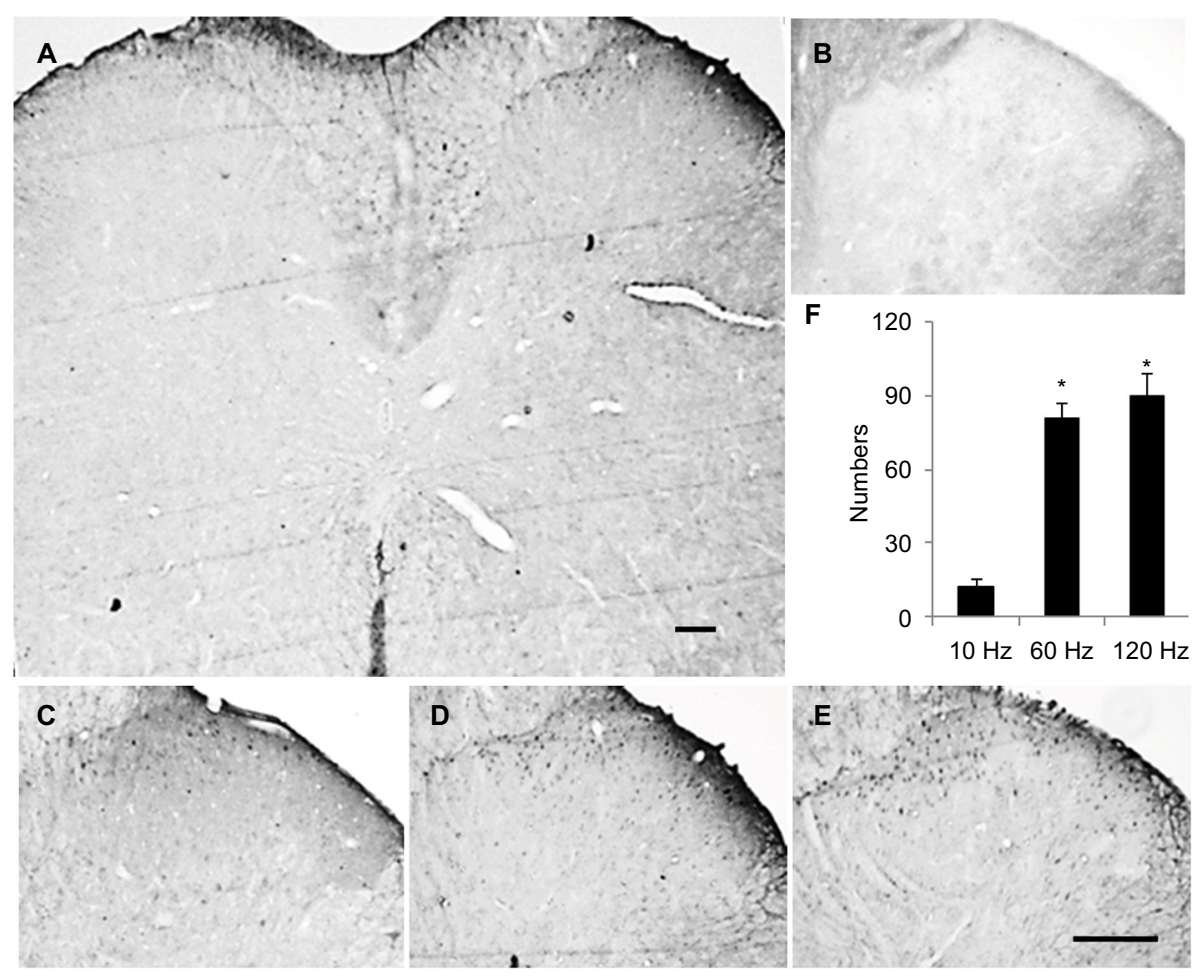

Figure 3 Localization of increasing Arc expression in spinal cord is detected with immunohistochemistry.

Notes: The results show that increasing Arc is located in superficial layers of spinal cord (A). (B) Negative control of Arc. Typical stains of Arc protein expression induced by PNS at $10 \mathrm{~Hz}(\mathbf{C}), 60 \mathrm{~Hz}(\mathbf{D})$, and $120 \mathrm{~Hz}(\mathbf{E})$ and the statistical results $(\mathbf{F})$. The Arc expression in sham (data not shown) is similar to that of $10 \mathrm{~Hz}$ PNS group. Bar $=100$ $\mu \mathrm{m}$. $\mathrm{n}$ of each group $=4 ; * P<0.0 \mathrm{I}$, compared with $10 \mathrm{~Hz}$ group. Data are presented as median \pm MAD and analyzed with Mann-Whitney $U$ test.

Abbreviations: PNS, peripheral nerve stimulation; MAD, median absolute deviation; Arc, activity-regulated cytoskeleton-associated protein.

A Sham $\quad \mathrm{TCl} \quad \mathrm{TCl}+60 \mathrm{~Hz} \mathrm{TCl}+120 \mathrm{~Hz} \mathrm{kDa}$
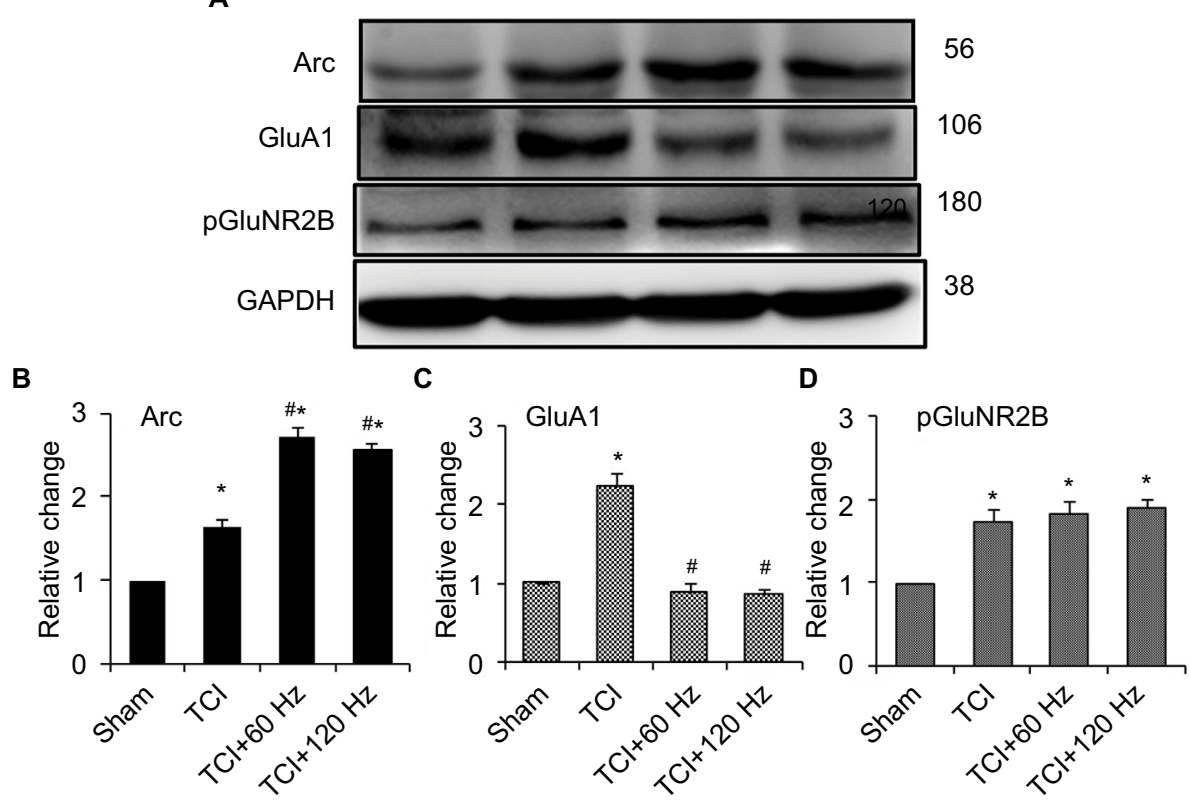

Figure 4 Effect of PNS on the expression of Arc, GluAI, and pGluNR2B in the spinal cord of bone cancer animal model.

Notes: Western blotting of Arc (A, B), GluAI (A, C), and pGluNR2B (A, D) reveals the effect of $60 \mathrm{~Hz}$ and I20 Hz PNS on its expression in the spinal cord. $n$ of each group $=5$; ${ }^{*} P<0.05$, compared with sham; ${ }^{*} P<0.05$, compared with $\mathrm{TCl}$ group. Data are presented as median \pm MAD and analyzed with Mann-Whitney $U$ test.

Abbreviations: GluAI, $\alpha$-amino-3-hydroxy-5-methyl-4-isoxazolepropionic acid-type glutamate receptor I; pGluNR2B, phosphate N-methyl-D-aspartic acid-type glutamate receptor subunit 2B; PNS, peripheral nerve stimulation; MAD, median absolute deviation; TCl, tumor cell implantation; Arc, activity-regulated cytoskeleton-associated protein. 


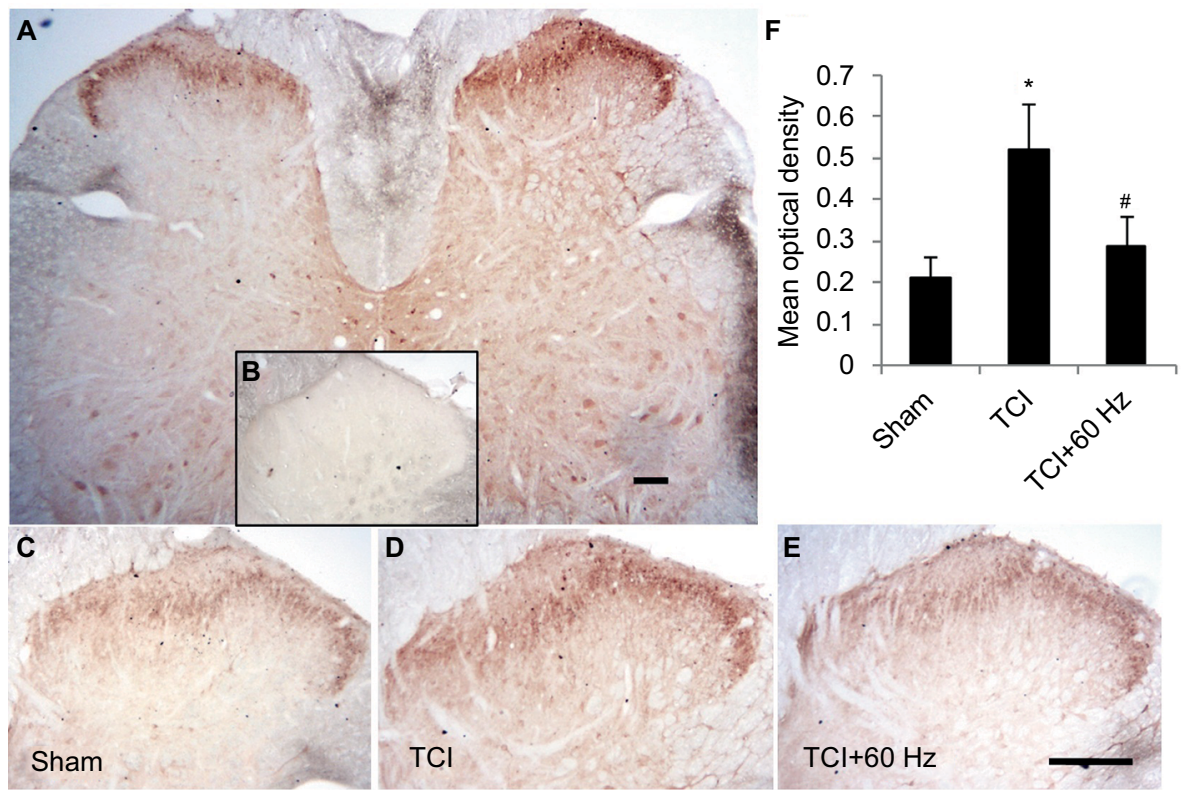

Figure 5 Increasing expression of GluAl in superficial layers of the spinal cord in $\mathrm{TCl}$ model is downregulated by $60 \mathrm{~Hz} \mathrm{PNS}$.

Notes: GluAI distributes in the entire spinal cord and is intensively expressed in neurons located in the superficial layers of dorsal horn (A). Negative control stain (B). GluAI expression in $\mathrm{TCl}(\mathbf{D})$ increases compared with control level (C), which is downregulated by $60 \mathrm{~Hz}$ PNS (E). Results of Statistic analysis was demonstrated (F). Bar $=100 \mu \mathrm{m}$. $n$ of each group $=5 ;{ }^{* P}<0.05$, compared with sham; ${ }^{*} P<0.05$, compared with TCl group. Data are presented as median \pm MAD and analyzed with Mann-Whitney $U$ test. Abbreviations: GluAI, $\alpha$-amino-3-hydroxy-5-methyl-4-isoxazolepropionic acid-type glutamate receptor I; PNS, peripheral nerve stimulation; MAD, median absolute deviation; $\mathrm{TCl}$, tumor cell implantation.

$60 \mathrm{~Hz}$ PNS on bone cancer pain was blunted (Figure 6A and B). Western blotting showed that Arc shRNA inhibited Arc expression, alleviating GluA1 induced by PNS (Figure 6C-E).

\section{Consistency testing between $\mathrm{TCl}$ group and $\mathrm{TCl}+0 \mathrm{~Hz}$ group}

TCI with $0 \mathrm{~Hz}$ PNS (sham stimulation) was exactly controlled for revealing the effect of PNS on bone cancer pain, while its function was replaced by TCI group in the rest experiments. To demonstrate whether conclusions were influenced by this condition, additional experiments were performed to test the consistency between TCI group and TCI+0 Hz group. The results showed that thermal hyperalgesia and mechanical allodynia behavior were similar between TCI group and $\mathrm{TCI}+0 \mathrm{~Hz}$ group (Figure S1A and S1B); expressions of Arc protein, GluA1, and pGluNR2B in spinal cord between the TCI group and TCI+0 Hz group did not exhibit statistical differences (Figure S1C-S1F).

\section{Discussion}

An indication of PNS is chronic or severe pain that undermines the functionality of patients and leaves them dependent on frequent medications, physical therapy, and less-invasive interventions, such as local anesthetic and sympathetic blocks, and transcutaneous electrical nerve stimulation. ${ }^{25}$ In this experiment, PNS alleviated intractable bone cancer pain, a typical indication of PNS, by inducing Arc expression in the spinal cord, which proves that PNS is effective in treating bone cancer pain.

Arc mediates PNS' effect on pain relief in two ways. First, PNS inducing Arc expression and PNS exerting an anti-nociceptive effect had the same parameters. Indeed, PNS at 60 or $120 \mathrm{~Hz}(0.3 \mathrm{~mA}, 20 \mathrm{~min})$ overtly induced Arc expression in spinal dorsal horn in naïve rats; PNS with the same parameters exhibited anti-nociceptive effect on both naïve and bone cancer pain states. However, low-frequency PNS $(10 \mathrm{~Hz}, 0.3 \mathrm{~mA}, 20 \mathrm{~min})$ failed to induce Arc expression in spinal dorsal horn and relieve bone cancer pain, and increase pain thresholds in naïve rats. In agreement with these findings, PNS at 40-100 Hz and low density showed its strong ability to relieve multiple types of pain in the clinic; ${ }^{10-12}$ meanwhile, $50 \mathrm{~Hz}$ PNS attenuated behavioral hypersensitivity to mechanical and thermal stimuli in nerve-injured rats. ${ }^{26}$ In this study, the effect of PNS at $>120 \mathrm{~Hz}$ was not explored because at that frequency it may induce reversible movement dysfunction resulting from motor nerve damage. Secondly, intrathecal administration of Arc shRNA inhibited the antinociceptive effect of PNS. Being able to silence Arc protein, ${ }^{27}$ Arc shRNA was administered 1 day before PNS to decrease PNS-induced Arc expression. These findings revealed that 


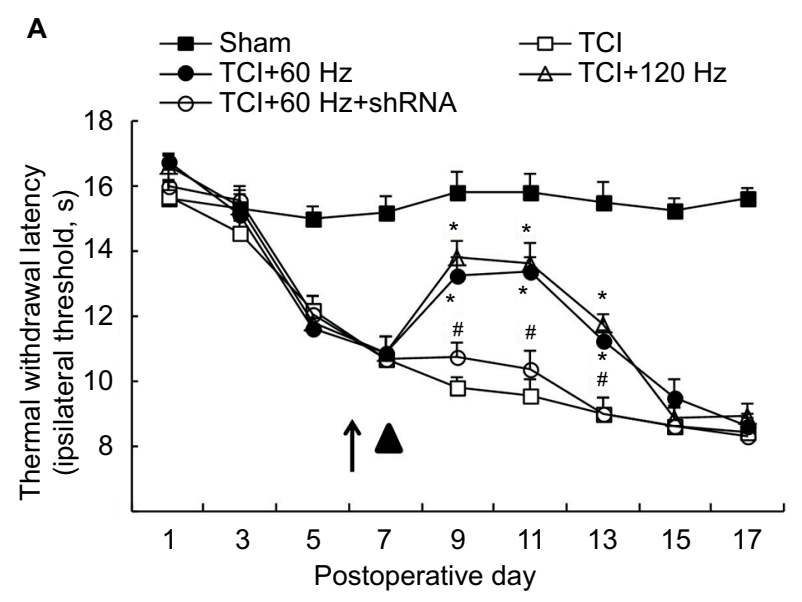

C

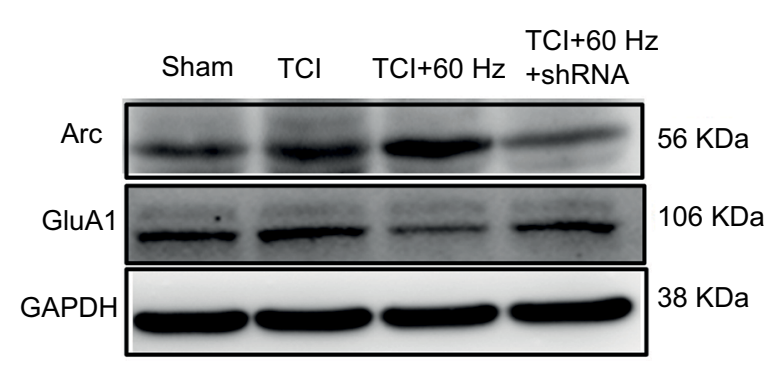

B
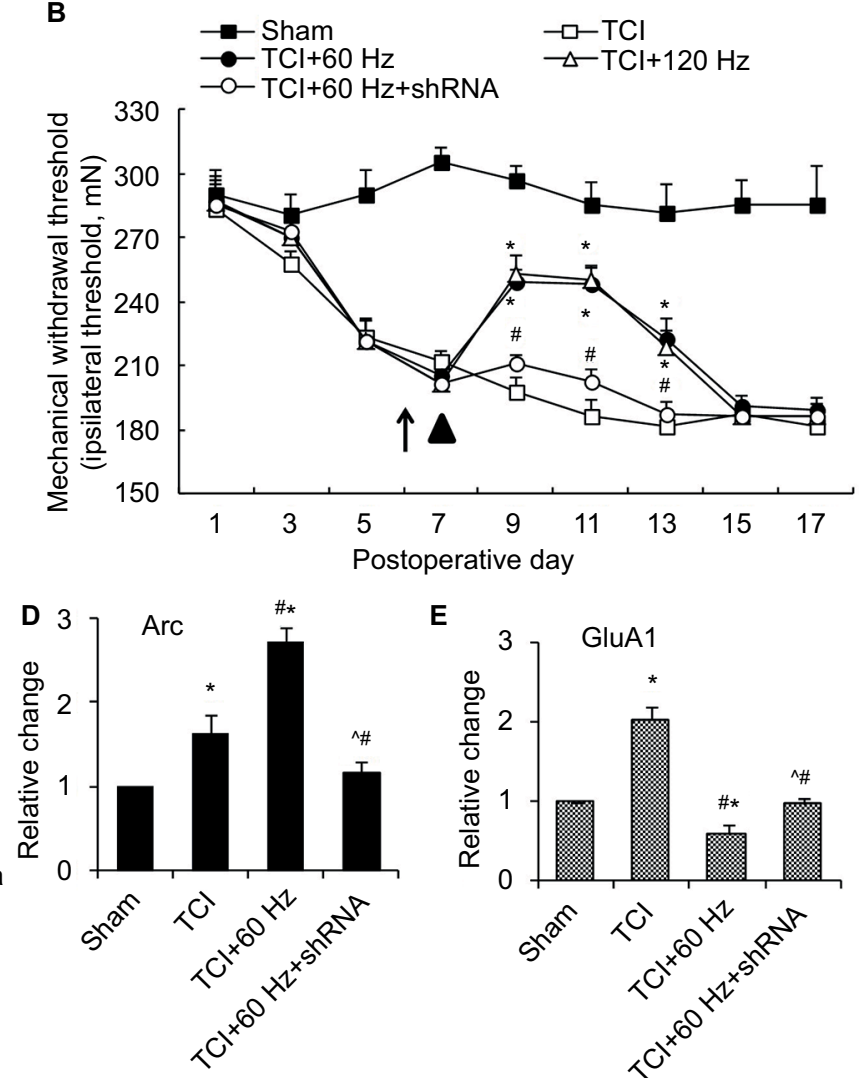

E

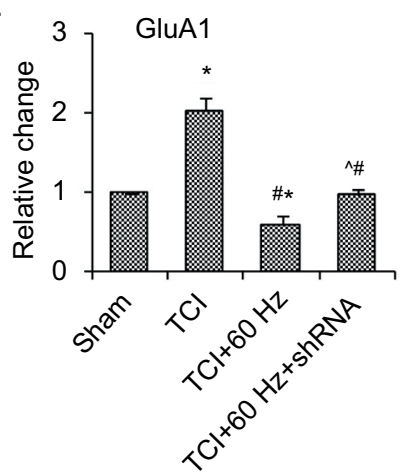

Figure 6 Arc shRNA regulates the anti-nociceptive effect of $60 \mathrm{~Hz}$ PNS and the expression of Arc and GluAI.

Notes: Tests of thermal withdrawal latency $(\mathbf{A})$ and mechanical withdrawal threshold $(\mathbf{B})$ were performed to reflect hyperalgesia and allodynia separately in the bone cancer model. PNS $(\mathbf{\Lambda})$ was performed on the 8th day after tumor cell implantation and Arc shRNA $(\uparrow)$ was intrathecally injected I day before PNS. $n$ of each group $=8$; $* P<0.05$, compared with sham; ${ }^{\# P}<0.05$, compared with $\mathrm{TCl}+60 \mathrm{~Hz}$ group. Data are presented as mean $\pm \mathrm{SEM}$ and analyzed with two-way ANOVA. As a result, Arc shRNA prohibits the expression of Arc induced by $60 \mathrm{~Hz}$ PNS as well as the reduction of GluAI (C-E). $n$ of each group $=5$; $* P<0.05$, compared with sham; ${ }^{*} P<0.05$, compared with TCl group; ${ }^{\wedge} P<0.05$, compared with $\mathrm{TCl}+60 \mathrm{~Hz}$ group. Data are presented as median \pm MAD and analyzed with Mann-Whitney $U$ test.

Abbreviations: ANOVA, analysis of variance; GluAI, $\alpha$-amino-3-hydroxy-5-methyl-4-isoxazolepropionic acid-type glutamate receptor I; PNS, peripheral nerve stimulation; MAD, median absolute deviation; TCl, tumor cell implantation; SEM, standard error of the mean; Arc, activity-regulated cytoskeleton-associated protein

Arc protein mediates the anti-nociceptive effect of PNS on bone cancer pain.

Arc expression induced by PNS relieves bone cancer pain by decreasing AMPAR transcription. It has been reported that Arc regulates AMPAR function through two mechanisms. First, numerous experiments have demonstrated the association between Arc and AMPAR internalization; ${ }^{19,28,29}$ Arc/Arg3.1 mRNA is transported to dendrites and translated at sites of high synaptic activity where its expression gets a temporal and spatial specificity. This protein then interacts with dynamin 2 and specific endophilin isoforms to localize endosomes and enhance AMPAR endocytosis in hippocampal neurons. ${ }^{17,28,29}$ Second, Arc decreases GluA1 transcription and synaptic strength. ${ }^{20}$ We observed that Arc increased and GluA1 phosphorylation decreased after PNS, simultaneously; meanwhile, Arc shRNA reversed the decrease of GluA1 expression induced by PNS. These findings indicate that PNS relieves bone cancer pain by decreasing the expression of AMPAR receptors. Expression of pGluNR2B was also observed after PNS in naïve animals and those with bone cancer pain. The fact that PNS did not influence pGluNR2B expression suggested that PNS selectively decreases GluA1 expression by inducing Arc protein expression in the spinal cord.

During PNS, it is necessary to demonstrate exactly which part of the nerve or what type of nerve fiber was stimulated. In our experiment, a bipolar electrode was placed on one side of the sciatic nerve, and not all nerve fibers were supposed to be stimulated directly. The results of immunochemistry showed that Arc expression increased by 60 and $120 \mathrm{~Hz}$ PNS was mainly distributed in lamina I and outer lamina II of the spinal cord, proving that peptidergic $\mathrm{C}$ fibers and nonpeptidergic $\mathrm{C}$ fibers were partly stimulated. But whether the other fibers, such as $\mathrm{A} \beta$ myelinated fibers and $\mathrm{A} \delta$ myelinated fibers, were stimulated simultaneously need to be further confirmed. Leg retraction was also observed, suggesting that the motor nerve 
fibers in sciatic nerves were stimulated by PNS. Besides, we also planned to identify the type of fibers stimulated by $60 \mathrm{~Hz}$ PNS with immunohistochemistry of activating transcription factor 3 in dorsal root ganglia (data not shown), which has been used to identify the affected fibers under $100 \mathrm{~Hz}$ PNS stimulation. ${ }^{30}$ However, we abandoned this method because false positive stain was observed on control DRG. At present, although we have no method to pinpoint the stimulated nerve fibers, we have proved according to our theory and clinical practice that the stimulation efficiency decides the analgesic effect. That is why flat and circumferential electrodes with higher stimulation efficiency have replaced bipolar electrodes in PNS. ${ }^{6,7}$ Our experiment provides the possibility of using PNS as an adjuvant treatment for bone cancer pain. However, multiple-site metastatic bone carcinoma with accompanying pain is more common and difficult to cure through PNS with a single electrode. With improved electrodes, such as electrode array, PNS might relieve multiple-site bone cancer pain.

Another interesting phenomenon is that PNS at the same frequency has the same effectiveness in relieving pain in both humans and rats. ${ }^{7,25,31}$ Therefore, once the Arc protein is identified as the mediator in the pain-relieving process, it can be used as a marker for PNS' effectiveness in treatment of chronic pain, help to develop the best PNS strategy for animals, and can be further applied to clinical treatment.

Currently, the most effective and widely used nerve stimulation method is $\mathrm{SCS} .{ }^{32}$ The recently developed DRGS also shows satisfactory outcomes in the treatment of intractable pain. ${ }^{33}$ Both SCS and DRGS stimulate the sensory nerve system without affecting the motor nerve system, which makes them effective in controlling intractable chronic pain with fewer side effects. It is too early to say that our findings are also true for SCS and DRGS; however, previous studies have revealed that SCS shares some characteristics with PNS. Both techniques can inhibit the windup in the wide dynamic range neurons of the spinal cord $^{34}$ and increase GABA concentrations. ${ }^{15}$ In addition, both use the same frequency in the treatment of pain. ${ }^{34}$ Therefore, there is a possibility that SCS and DRGS could relieve pain by inducing Arc expression and decreasing AMPAR expression, which will be explored in future research. With the development of advanced techniques, including voltage-controlled capacitive discharge enabling wireless neuromodulation ${ }^{26}$ and less-invasive ultrasound-guided implantation technique, ${ }^{35}$ neuromodulation could be expanded in clinic.

If the results and theory in this article were applied in clinical practice, differences between bone cancer pain of patients and animals should be noticed. A significant point is that the pain (hyperalgesia and allodynia) of animals is induced, but the pain of patients is spontaneously felt and evaluated in clinic. Some researchers believe that this difference could be responsible for the translation failure of several medicines that are effective in animals, but not in patients. ${ }^{36}$ So our findings should be confirmed by future experiments that focus on animal spontaneous pain assessed with a good evaluation system.

\section{Conclusion}

PNS at 60 and $120 \mathrm{~Hz}(0.3 \mathrm{~mA})$ can relieve bone-cancerinduced allodynia and hyperalgesia by increasing Arc expression and decreasing AMPAR transcription in the spinal cord in an animal model.

\section{Abbreviations}

PBS, phosphate-buffered saline; PNS, peripheral nerve stimulation; Arc protein, activity-regulated cytoskeleton-associated protein; GluA1, $\alpha$-amino-3-hydroxy5-methyl-4-isoxazolepropionic acid-type glutamate receptor 1; pGluNR2B, phosphate $N$-methyl-D-aspartic acid-type glutamate receptor subunit $2 \mathrm{~B}$; TENS, transcutaneous electrical nerve stimulation; PENS, percutaneous peripheral electrical nerve stimulation; GABA, $\gamma$-aminobutyric acid; AMPAR, $\alpha$-amino-3-hydroxy-5methyl-4-isoxazolepropionic acid-type glutamate receptor; SCS, spinal cord stimulation; TCI, tumor cell implantation; H-E, hematoxylin and eosin; ANOVA, analysis of variance; SEM, standard error of the mean; PWTL, paw withdrawal thermal latency; PWMT, paw withdrawal mechanical thresholds; DRG, dorsal root ganglion; DRGS, dorsal root ganglion stimulation.

\section{Acknowledgments}

We thank Dr Xue-jun Song for providing Walker 256 cells as a gift, Prof Xue-ming He for general assistance, and $\mathrm{Su}$ Liu for technical support in the making of the bone cancer pain animal model. Both Xue-ming $\mathrm{He}$ and $\mathrm{Su}$ Liu are employees of the Key Lab of Anesthesiology of Xuzhou Medical University.

This work was supported by the Lianyungang Municipal Science and Technology Bureau Foundation (SH1339, SH1544, and SH1545).

\section{Author contributions}

Ke-fu Sun and Hui-lin Yang designed this experiment and wrote the article. Ke-fu Sun, Wan-wen Feng, Yue-peng Liu, Yan-bin Dong, and Li Gao performed data acquisition and data analysis. All authors contributed toward data analysis, 
drafting and revising the paper and agree to be accountable for all aspects of the work.

\section{Disclosure}

The authors report no conflicts of interest in this work.

\section{References}

1. Breivik H, Cherny N, Collett B, et al. Cancer-related pain: a panEuropean survey of prevalence, treatment, and patient attitudes. Ann Oncol. 2009;20(8):1420-1433.

2. Mercadante S, Fulfaro F. Management of painful bone metastases. Curr Opin Oncol. 2007;19(4):308-314.

3. Lynch TN KJ, Vasudevan SV. Psychological Examinations and Psychometric Testing. Practical Management of Pain. 2nd ed. St Louis: Mosby Year Book; 1992.

4. Manchikanti L, Ailinani H, Koyyalagunta D, et al. A systematic review of randomized trials of long-term opioid management for chronic noncancer pain. Pain Physician. 2011;14(2):91-121.

5. Vance CG, Dailey DL, Rakel BA, Sluka KA. Using TENS for pain control: the state of the evidence. Pain Manag. 2014;4(3):197-209.

6. Alo KM, Abramova MV, Richter EO. Percutaneous peripheral nerve stimulation. Prog Neurol Surg. 2011;24:41-57.

7. Reverberi C, Dario A, Barolat G, Zuccon G. Using peripheral nerve stimulation (PNS) to treat neuropathic pain: a clinical series. Neuromodulation. 2014;17(8):777-783; discussion 783.

8. Huntoon MA, Burgher AH. Ultrasound-guided permanent implantation of peripheral nerve stimulation (PNS) system for neuropathic pain of the extremities: original cases and outcomes. Pain Med. 2009;10(8):1369-1377.

9. Thakral G, Kim PJ, LaFontaine J, Menzies R, Najafi B, Lavery LA. Electrical stimulation as an adjunctive treatment of painful and sensory diabetic neuropathy. J Diabetes Sci Technol. 2013;7(5):1202-1209.

10. Lee PB, Horazeck C, Nahm FS, Huh BK. Peripheral nerve stimulation for the treatment of chronic intractable headaches: long-term efficacy and safety study. Pain Physician. 2015;18(5):505-516.

11. Stidd DA, Wuollet AL, Bowden K, et al. Peripheral nerve stimulation for trigeminal neuropathic pain. Pain Physician. 2012;15(1):27-33.

12. Stevanato G, Devigili G, Eleopra R, et al. Chronic post-traumatic neuropathic pain of brachial plexus and upper limb: a new technique of peripheral nerve stimulation. Neurosurg Rev. 2014;37(3):473-479; discussion 479-480.

13. Rauck RL, Kapural L, Cohen SP, et al. Peripheral nerve stimulation for the treatment of postamputation pain - a case report. Pain Pract. 2012;12(8):649-655.

14. Melzack R, Wall PD. Pain mechanisms: a new theory. Science. 1965;150(3699):971-979.

15. Somers DL, Clemente FR. Contralateral high or a combination of highand low-frequency transcutaneous electrical nerve stimulation reduces mechanical allodynia and alters dorsal horn neurotransmitter content in neuropathic rats. J Pain. 2009;10(2):221-229.

16. Song Z, Meyerson BA, Linderoth B. Spinal 5-HT receptors that contribute to the pain-relieving effects of spinal cord stimulation in a rat model of neuropathy. Pain. 2011;152(7):1666-1673.

17. Bramham CR, Worley PF, Moore MJ, Guzowski JF. The immediate early gene arc/arg3.1: regulation, mechanisms, and function. JNeurosci. 2008;28(46):11760-11767.
18. Waung MW, Pfeiffer BE, Nosyreva ED, Ronesi JA, Huber KM. Rapid translation of Arc/Arg3.1 selectively mediates mGluR-dependent LTD through persistent increases in AMPAR endocytosis rate. Neuron. 2008;59(1): 84-97.

19. Shepherd JD, Rumbaugh G, Wu J, et al. Arc/Arg3.1 mediates homeostatic synaptic scaling of AMPA receptors. Neuron. 2006;52(3):475-484.

20. Korb E, Wilkinson CL, Delgado RN, Lovero KL, Finkbeiner S. Arc in the nucleus regulates PML-dependent GluA1 transcription and homeostatic plasticity. Nat Neurosci. 2013;16(7):874-883.

21. Tao YX. Dorsal horn alpha-amino-3-hydroxy-5-methyl-4-isoxazolepropionic acid receptor trafficking in inflammatory pain. Anesthesiology. 2010;112(5):1259-1265.

22. Su C, D'Amour J, Lee M, et al. Persistent pain alters AMPA receptor subunit levels in the nucleus accumbens. Mol Brain. 2015;8:46.

23. Hossaini M, Jongen JL, Biesheuvel K, Kuhl D, Holstege JC. Nociceptive stimulation induces expression of Arc/Arg3.1 in the spinal cord with a preference for neurons containing enkephalin. Mol Pain. 2010;6:43.

24. Zhu GQ, Liu S, He DD, Liu YP, Song XJ. Activation of the cAMP-PKA signaling pathway in rat dorsal root ganglion and spinal cord contributes toward induction and maintenance of bone cancer pain. Behav Pharmacol. 2014;25(4):267-276.

25. Slavin KV. Peripheral nerve stimulation for neuropathic pain. Neurotherapeutics. 2008;5(1):100-106.

26. Rosellini W, Casavant R, Engineer N, et al. Wireless peripheral nerve stimulation increases pain threshold in two neuropathic rat models. Exp Neurol. 2012;235(2):621-626.

27. Liu Y, Zhou QX, Hou YY, et al. Actin polymerization-dependent increase in synaptic Arc/Arg3.1 expression in the amygdala is crucial for the expression of aversive memory associated with drug withdrawal. J Neurosci. 2012;32(35):12005-12017.

28. Chowdhury S, Shepherd JD, Okuno H, et al. Arc/Arg3.1 interacts with the endocytic machinery to regulate AMPA receptor trafficking. Neuron. 2006;52(3):445-459.

29. Rial Verde EM, Lee-Osbourne J, Worley PF, Malinow R, Cline HT. Increased expression of the immediate-early gene arc/arg3.1 reduces AMPA receptor-mediated synaptic transmission. Neuron. 2006;52(3): $461-474$.

30. Liang L, Wang Z, Lu N, Yang J, Zhang Y, Zhao Z. Involvement of nerve injury and activation of peripheral glial cells in tetanic sciatic stimulation-induced persistent pain in rats. JNeurosci Res. 2010;88(13): 2899-2910.

31. Henderson JM. Peripheral nerve stimulation for chronic pain. Curr Pain Headache Rep. 2008;12(1):28-31.

32. Wolter T. Spinal cord stimulation for neuropathic pain: current perspectives. J Pain Res. 2014;7:651-663.

33. DeerTR, Grigsby E, Weiner RL, Wilcosky B, Kramer JM. A prospective study of dorsal root ganglion stimulation for the relief of chronic pain. Neuromodulation. 2013;16(1):67-71; discussion 70.

34. Yang F, Xu Q, Cheong YK, et al. Comparison of intensity-dependent inhibition of spinal wide-dynamic range neurons by dorsal column and peripheral nerve stimulation in a rat model of neuropathic pain. Eur $J$ Pain. 2014;18(7):978-988.

35. Gofeld M, Hanlon JG. Ultrasound-guided placement of a paddle lead onto peripheral nerves: surgical anatomy and methodology. Neuromodulation. 2014;17(1):48-53; discussion 53.

36. Clark JD. Preclinical pain research: can we do better? Anesthesiology. 2016;125(5):846-849. 


\section{Supplementary material}

A
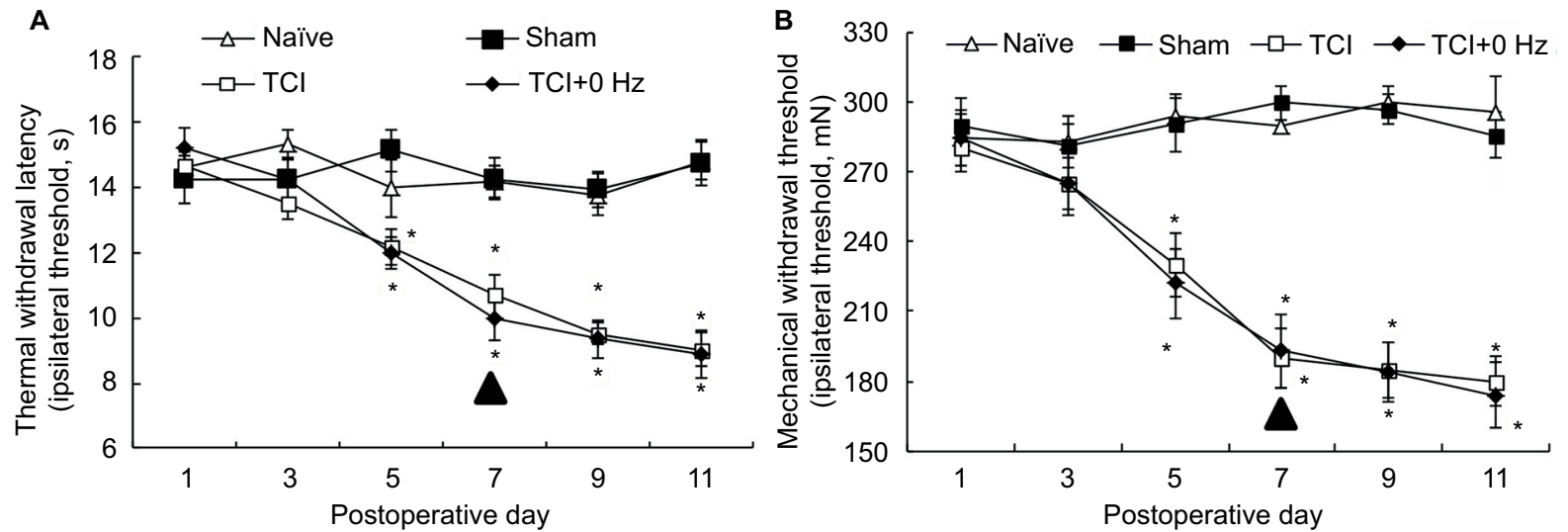

C

Naïve Sham

$\mathrm{TCl}$

$\mathrm{TCl}+0 \mathrm{~Hz}$

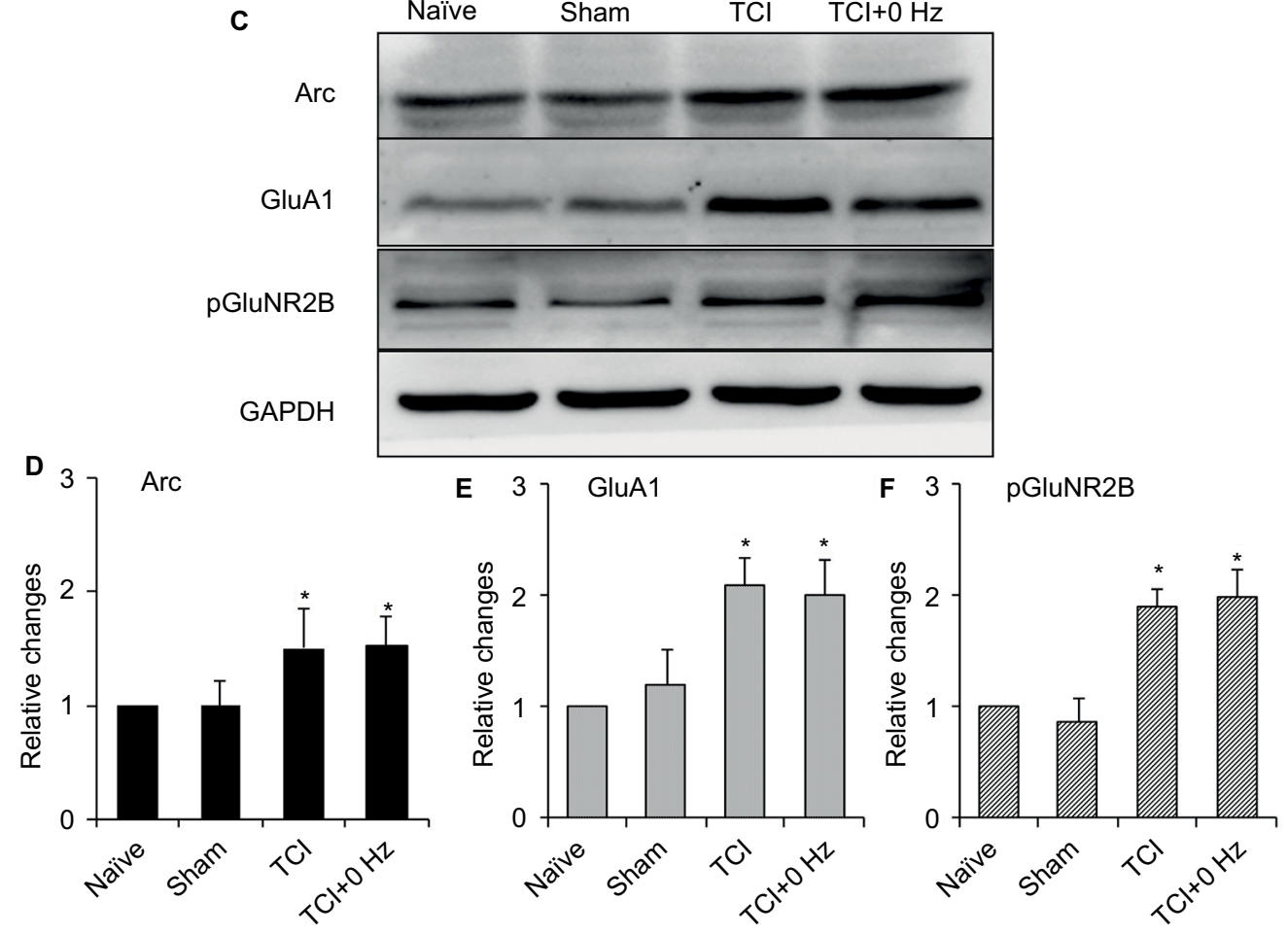

Figure $\mathbf{S I}$ Consistency testing between $\mathrm{TCl}$ group and $\mathrm{TCl}+0 \mathrm{~Hz}$ group.

Notes: (A) Thermal hyperalgesia; (B) mechanical allodynia ( $\mathrm{n}$ of each group =8; data are presented as mean \pm SEM and analyzed with two-way ANOVA); (C) typical Western blotting result of Arc protein, GluAI, and pGluNR2B; statistical results of (D) Arc protein, (E) GluAI, and $(\mathbf{F}) \mathrm{pGluNR2B}$. $* P<0.05$, compared with sham. $n$ of each group $=5$; data are presented as median \pm MAD and analyzed with Mann-Whitney $U$ test. PNS $(\boldsymbol{\Delta})$ was performed on day 0.

Abbreviations: ANOVA, analysis of variance; GluAI, $\alpha$-amino-3-hydroxy-5-methyl-4-isoxazolepropionic acid-type glutamate receptor I; pGluNR2B, phosphate N-methyl$\mathrm{D}$-aspartic acid-type glutamate receptor subunit 2B; MAD, median absolute deviation; PNS, peripheral nerve stimulation; TCI, tumor cell implantation; SEM, standard error of the mean; Arc, activity-regulated cytoskeleton-associated protein.

\section{Journal of Pain Research}

\section{Publish your work in this journal}

The Journal of Pain Research is an international, peer reviewed, open access, online journal that welcomes laboratory and clinical findings in the fields of pain research and the prevention and management of pain. Original research, reviews, symposium reports, hypothesis formation and commentaries are all considered for publication.

\section{Dovepress}

Submit your manuscript here: https://www.dovepress.com/journal-of-pain-research-journal

The manuscript management system is completely online and includes a very quick and fair peer-review system, which is all easy to use. Visit http://www.dovepress.com/testimonials.php to read real quotes from published authors. 